\title{
ON THE ASYMPTOTIC EXPANSION OF AIRY'S INTEGRAL
}

\author{
by E. T. COPSON
}

(Received 19 November, 1962)

1. Introduction. The integral function

$$
A i(z)=\frac{1}{3^{3} \pi} \sum_{0}^{\infty} \frac{\Gamma\left(n+\frac{1}{3}\right)}{n !} \sin \frac{2}{3}(n+1) \pi \cdot\left(3^{\frac{1}{3}} z\right)^{n}
$$

is known as Airy's Integral since, when $z$ is real, it is equal to the integral

$$
\frac{1}{\pi} \int_{0}^{\infty} \cos \left(\frac{1}{3} t^{3}+z t\right) d t
$$

which first arose in Airy's researches on optics. It is readily seen that $w=A i(z)$ satisfies the differential equation $d^{2} w / d z^{2}=z w$, an equation which also has solutions $A i(\omega z), A i\left(\omega^{2} z\right)$, where $\omega$ is the complex cube root of unity, $\exp \frac{2}{3} \pi i$. The three solutions are connected by the relation

$$
A i(z)+\omega A i(\omega z)+\omega^{2} A i\left(\omega^{2} z\right)=0 .
$$

Instead of using $A i(\omega z)$ or $A i\left(\omega^{2} z\right)$ as second solution, the function

$$
B i(z)=i \omega^{2} A i\left(\omega^{2} z\right)-i \omega A i(\omega z),
$$

which is real when $z$ is real, is commonly employed.

If we write $z=v^{2}$, it follows from (1.2) that, when $v>0$,

$$
A i\left(v^{2}\right)=\frac{1}{2 \pi i} \int_{I} e^{v^{2} s-t s^{3}} d s,
$$

where $I$ is the imaginary axis from $-\infty i$ to $\infty i$. By Cauchy's Theorem, the path can be replaced by any path $L$, such as a pair of radii, from $\infty \exp \frac{4}{3} \pi i$ to $\infty \exp \frac{2}{3} \pi i$, and the resulting integral represents $A i\left(v^{2}\right)$, not merely for $v>0$, but for all values of $\mathrm{ph} v$.

When $v$ is positive, it is convenient to make the change of variable $s=v w$, which gives

$$
A i\left(v^{2}\right)=\frac{v}{2 \pi i} \int_{C} e^{v^{3}\left(w-\frac{1}{2} w^{3}\right)} d w,
$$

where $C$ is the path $I$ or a path $L$. The integrand has saddle points $w= \pm 1$; and the path of steepest descents through $w=-1$ is $3 u^{2}-v^{2}=3$ (if we write $w=u+i v$ ), and this is a path $L$. The asymptotic expansion of $A i\left(v^{2}\right)$ for large $v$ when $v>0$, or, more generally, when $|\operatorname{ph} v|$ $<\frac{1}{6} \pi$ is obtainable by integrating along this hyperbolic path. The discussion is straight- 
forward but rather tedious. $\dagger$ It gives the asymptotic expansion of $A i(z)$ when $|\mathrm{ph} z|<\frac{1}{3} \pi$.

By a similar argument, the asymptotic expansion can be found when $|\mathrm{ph}(-z)|<\frac{1}{3} \pi$. To fill in the gap between these two angles is difficult.

It is the purpose of this note to show that the asymptotic expansion of $\operatorname{Ai}(z)$ when $|\mathrm{ph} z|<\pi$ can be obtained by a very much simpler argument, and that the asymptotic expansions of $A i(z)$ and $B i(z)$ for all values of $\mathrm{ph} z$ can be readily deduced by using formulae (1.3) and (1.4).

2. Another integral representation of $A i\left(v^{2}\right)$. We suppose first that $v>0$ and start from the formula (1.5). The point in the $s$ plane corresponding to the saddle point $w=-1$ is $s=-v$. We show that the integral is unaltered in value if the path $I$ is deformed into a parallel line through $s=-v$. By Cauchy's Theorem, all we have to show is that the integral along the st raight line from $s=-v+i t$ to $s=i t$ tends to zero as $t \rightarrow \pm \infty$.

Writing $s=\sigma+i t$, we have then to show that

$$
\int_{-v}^{0} e^{v^{2}(\sigma+i t)-3(\sigma+i t)^{3}} d \sigma
$$

tends to zero. The absolute value of this integral does not exceed

$$
\int_{-v}^{0} e^{v v^{2} \sigma-t \sigma^{3}+\sigma t^{2}} d \sigma \leqq e^{\frac{1}{v^{3}}} \int_{-v}^{0} e^{\sigma t^{2}} d \sigma<\frac{e^{+v^{3}}}{t^{2}}
$$

which tends to zero as $t \rightarrow \pm \infty$.

We may therefore put $s=-v+i t$ in (1.5), where $t$ varies from $-\infty$ to $+\infty$. This gives

or

$$
\begin{aligned}
& A i\left(v^{2}\right)=\frac{1}{2 \pi} e^{-z v^{3}} \int_{-\infty}^{\infty} e^{-v t^{2}+t i t^{3}} d t \\
& A i\left(v^{2}\right)=\frac{1}{\pi} e^{-z v^{3}} \int_{0}^{\infty} e^{-v t^{2}} \cos \left(\frac{1}{3} t^{3}\right) d t
\end{aligned}
$$

when $v>0$.

Before we apply Watson's Lemma, we observe that, when $v$ is complex, the integral (2.1) converges uniformly in $0<v_{0} \leqq|v| \leqq v_{1},|\mathrm{ph} v| \leqq \frac{1}{2} \pi-\delta<\frac{1}{2} \pi$ by Weierstrass's M-test; and as $A i\left(v^{2}\right)$ is an integral function of $v$, it follows that (2.1) holds in the half-plane $|\mathrm{ph} v|<\frac{1}{2} \pi$.

If we now write $t^{2}=u$, we have

$$
A i\left(v^{2}\right)=\frac{1}{2 \pi} e^{-\frac{3}{3} v^{3}} \int_{0}^{\infty} e^{-v u} \cos \left(\frac{1}{3} u^{\frac{3}{2}}\right) \frac{d u}{\sqrt{ } u} .
$$

As the conditions of Watson's Lemma are evidently satisfied, it follows at once that

† This steepest descents proof is due to Brillouin, Ann. Sci. de l'École Norm. Sup. 33 (1910), $17-69$. 


$$
A i\left(v^{2}\right) \sim \frac{1}{2 \pi} e^{-3 v^{3}} \sum_{0}^{\infty} \frac{\Gamma\left(3 n+\frac{1}{2}\right)}{3^{2 n}(2 n) !} \frac{(-1)^{n}}{v^{3 n+\frac{1}{2}}},
$$

and hence that

$$
A i(z) \sim \frac{1}{2 \pi z^{\frac{3}{4}}} e^{-\frac{z z}{2}} \sum_{0}^{\infty} \frac{\Gamma\left(3 n+\frac{1}{2}\right)}{3^{2 n}(2 n) !} \frac{(-1)^{n}}{z^{\frac{2}{3 n}}}
$$

when $|\operatorname{ph} z|<\pi$.

It will be observed that this proof is not only simpler than that of Brillouin; it also gives a much better result.

3. Extension of the range of values of $\mathrm{ph} z$.

The result just proved holds in any angle not containing the negative real axis. To extend the range of values of $\mathrm{ph} z$, we use equation (1.3), viz.

$$
\operatorname{Ai}(z)=-\omega A i(\omega z)=\omega^{2} A i\left(\omega^{2} z\right)
$$

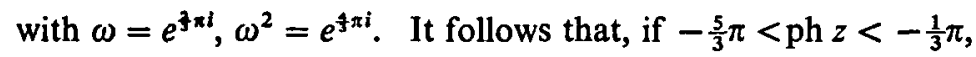

$$
\operatorname{Ai}(z) \sim F(z)-i G(z),
$$

where

$$
\begin{aligned}
& F(z) \sim \frac{1}{2 \pi z^{\frac{\pi}{4}}} e^{-3 z^{\frac{z}{2}}} \sum_{0}^{\infty} \frac{\Gamma\left(3 n+\frac{1}{2}\right)}{3^{2 n}(2 n) !} \frac{(-1)^{n}}{z^{\frac{3}{3^{n}}}}, \\
& G(z) \sim \frac{1}{2 \pi z^{\frac{3}{4}}} e^{3 z^{\frac{1}{2}}} \sum_{0}^{\infty} \frac{\Gamma\left(3 n+\frac{1}{2}\right)}{3^{2 n}(2 n) !} \frac{1}{z^{\frac{2}{n}}} .
\end{aligned}
$$

But if we take $\omega=e^{-\frac{4}{3} n i}, \omega^{2}=e^{-\frac{3}{3} \pi i}$, we find that

$$
A i(z) \sim F(z)+i G(z)
$$

when $\frac{1}{3} \pi<\operatorname{ph} z<\frac{5}{3} \pi$.

We have thus obtained three different asymptotic expansions, namely $F(z)$ and $F(z) \pm i G(z)$, for the integral function $A i(z)$ valid in three overlapping angles. The expansions are consistent, the change of form as ph $z$ varies being well known-it is the Stokes Phenomenon.

In a similar way, we can obtain asymptotic expansions for $B i(z)$ by using (1.4); but as the results are well known, we shall not discuss them further.

St Salvator's College

UNIVERSITY OF ST ANDREWS 